\title{
Preoperative Evaluation of Thyroid Nodules: A Prospective Study Comparing the accuracy of Ultrasound (TI-RADS) Versus the FNAC Bethesda System in Relation to the Final Postoperative Histo-pathological Diagnosis
}

\author{
Ashraf M Abdelkader ${ }^{1 *}$, Ahmed Mohamed Zidan ${ }^{1}$, Mohamed Tawfeek Younis ${ }^{1}$, Shaimaa Kamal Dawa ${ }^{2}$ \\ ${ }^{1}$ General surgery department, Faculty of medicine, Benha University, EGYPT \\ ${ }^{2}$ Pathology Department, Faculty of Medicine, Benha University, EGYPT
}

\begin{abstract}
Objectives: We are trying to improve and detect the accuracy of the diagnostic tools of thyroid nodules by comparing the findings of thyroid ultrasound (US) using the thyroid image reporting and data system (TI-RADS) with the results of fine needle aspiration cytology (FNAC) that were reported according to the Bethesda system for reporting thyroid Cytopathology (TBSRTC), through matching the results of both maneuverswith the final postoperative (PO)pathology reports.

Methods:The study included 100 patients suffering from thyroid swelling.Patients underwent ultrasound assessment using TI-RADS and FNAC biopsy using TBSRTCand then, all patients underwent thyroidectomy operation. Specimens sent to a laboratory for histological examination. The results of TI-RADS compared with Bethesda categories, and then both results were matched with the final histology reports. Data collected and statisticallyanalyzed.

Results: The overall concordance rate between US TI-RADS and TBSRTC is $67.6 \%$. ( $82 \%$ in benign cases, $70.9 \%$, in indeterminate cases, $50 \%$ in malignant cases). The overall concordance rate of results of TI-RADS versus FNAC with the final PO pathological results for predicting malignancy were $(75.4 \%, 81.8 \%)$ with a sensitivity of $(76.9 \%, 81.8 \%)$ and specificity of $(91.3 \%, 98 \%)$, positive predictive valueswere (PPV)(71.4\%,90\%), and negative predictive values were (NPV)(76.4\%, 96\%), respectively.

Conclusion: TI-RADS and TBSRTC classification systems could be considered as feasible and effective diagnosticmodalities for predicting malignant lesions in patients had thyroid nodules. It's important for the clinicians to implement these diagnostic tests to improve their clinical performance and surgical outcomes.
\end{abstract}

Keywords: Thyroid nodules, TI-RADS, Bethesda system, Thyroidectomy.

\section{Introduction}

The incidence of thyroid nodules is $2-6 \%$ with clinical examination, 19-68\% with ultrasound (US), and in autopsy specimens, it is $8-65 \% .^{[1,2,3]}$ Thyroid nodules are 4 times more common in females than in males and more frequent in the iodine-deficient areas. Thyroid nodules' management has become increasingly a subject of debate with the increasing frequency of thyroid malignancies. Thyroid nodules can be (1) benign (Hashimoto's thyroiditis, subacute thyroiditis, colloid nodule, simple cyst, hemorrhagic cyst, and follicular adenoma) or (2) malignant (Papillary, Follicular, Medullary, Anaplastic, Hurthle Cell (oncocytic) carcinomas, thyroid lymphoma and metastases). ${ }^{[4,5]}$

Evaluation of a patient with a thyroid nodule should be initially started with a detailed history, clinical examination, Laboratory exams, and imaging studies. ${ }^{[6]}$ Ultrasound is a noninvasive radiological technique that should be done in all patients with a thyroid disease to confirm the nodule/s, measure the size, site and assess the echogenicity, composition, shape, margins, vascularity, and the presence of calcification in the nodules as well as evaluate the other structures in the neck comprising the lymph nodes. ${ }^{[7,8,9]}$ FNAC biopsy is the next step in the assessment of thyroid nodules. ${ }^{[10]}$ According to the American Association of Clinical Endocrinologists guidelines, FNAC biopsy is "the most effective and accessible method for differentiating between malignant and benign thyroid lesions," with an accuracy around $95 \% .{ }^{[11]}$ In this study, we are trying to evaluate and compare the accuracy of both TI-RADS versus TBSRTC in the evaluation of thyroid nodules.

\section{Patients and Methods}

The present study conducted at the General Surgery and Pathology departments, Benha University Hospital in Egypt and King Saud Hospital in Saudi Arabia from 
January 2016 until January 2018. The study includes 100 consecutive patients with a clinically palpable thyroid nodule/nodules. After approval of the study protocol by the Ethical Committee and obtaining fully informed written patients' consent for the participation in the study.

Patients evaluated in the OPD through clinical, laboratory and radiological tools. Detailed medical history recorded, then patients examined generally plus local neck examination. Laboratory exams are done such as serumfree triiodothyronine (T3), free tetraiodothyronine (T4), thyroid-stimulating hormone (TSH), and Serum calcitonin. Diagnostic imaging was done by means of (1) radionuclide thyroid scan/scintigraphy, which was done in patients suffering from a thyroid nodule with low serum TSH to exclude the possibility of subclinical hyperthyroidism. (2) Thyroid US that was scored according to TIRADS classification as follows: (1) normal thyroid gland, (2) benign lesions, (3) probably benign lesions, (4) suspicious lesions, (5) probably malignant lesions, and (6) biopsy-proven malignancy. ${ }^{[12]}$ The FNAC was implemented for all patients with a TIRADS $>2$. Assessment of FNAC was done according to TBSRTC: class (I) non-diagnostic or unsatisfactory, (II) benign, (III) atypia of undetermined significance (AUS) or follicular lesion of undetermined significance (FLUS); (IV) follicular neoplasm or suspicious for a follicular neoplasm, (V) suspicious for malignancy, and (VI) malignant. ${ }^{[13]}$ Before surgery, patients were admitted to the General Surgery Department and prepared for surgery, then operation done after signing a fully informed separate consent for the operation. Exclusion criteria were: patients with TI-RADS $1 \& 2$ and nodules less than $1 \mathrm{~cm}$ with no US feature suspicious for malignancy (no indication for FNAC or surgery), former thyroid or parathyroid operations, previous thyroid FNAC in the last 3 months (FNAC not recommended), unsatisfactory FNAC sample, age $<18$ years, patients' inability to offer informed consent.

Thyroid US Technique: The Real-time B - mode US and Doppler assessment of the thyroid nodules was done by a radiologist with a good practice in thyroid US. The US scans were completed on GE VOLUSON 730 PRO machine (GE Healthcare, Milwaukee, USA) with a highfrequency linear array transducer $(7.5-12 \mathrm{MHz})$. Images were inspected on the real-time two-dimensional grayscale and Doppler imaging. Patient placed in the supine position and hyper-extended neck. The neck was scanned in transverse, sagittal, and oblique sections to ideally see both thyroid lobes, isthmus. Also, the regions of the jugular veins and carotid arteries, as well as the supra-clavicular fossa, were visualized for any lymph node enlargement. The sonographic features in all saved images were examined; such as the internal composition, echogenicity, margins, presence of calcifications, and the shape of the nodule (Fig. 1). For a patient with more than one thyroid nodule, classified as multinodular goiter (MNG) and the nodule with the most suspicious sonographic features was recorded as the nodule of interest. The internal structure of the nodule demarcated as cystic, solid, or mixed. The US parameters defined the suspicion of malignancy as follows: irregular border, micro-calcifications, hypo-echogenicity, and central flow by Doppler study. The nodule was defined as probably benign If it did not show suspicious features of malignancy.

The Technique of US-guided FNAC Biopsy: All procedures were performed by the pathologist and the radiologist, after obtaining a written informed consent, the patient was placed in the supine position with the hyper-extended neck. The skin and the 7.5-12 MHz linear transducer were cleansed with Betadine (povidone-iodine) solution. A sterile gel used as a coupling agent. The Initial US study was done to recognize the nodule of interest that indicated for FNAC according to the guidelines (Fig. 2). Then, the Tyco Healthcare Monoject non-cutting bevelled edge needle (25-gauge, $5 \mathrm{~cm}$ in length) (Sherwood Medical, St. Louis, MO) was held in the right hand and the transducer in the left hand. The needle was connected to a $10 \mathrm{ml}$ syringe, then it was entered through the skin at an oblique angle under US guidance, then it was moved through the centre of the nodule gently and rapidly with a mild suction. If the aspirated fluid contained much blood, a non-aspiration technique was applied, and the needle was moved in back and forth directions. A hematoma excluded through a post-procedure US.

Specimen Preparation and Interpretation: By the pathologist, the aspirate was expelled over two clean slides ( 2 drops for each slide) and spread to form a film by a clean slide at $60^{\circ}$ angle. Both slides were placed in ethanol $95 \%$ for fixation, then stained via the modified Papanicolaou procedure, and then submitted for evaluation and categorized into 1 of 6 categories according to the classification of the Bethesda system (Fig. 4). ${ }^{[13]}$

Surgery: Surgery was done for all patients included in our study. Patients with lesions suspicious of papillary or medullary thyroid cancer underwent a neck US \pm CT imaging to evaluate for any metastatic lesions. The US \pm CT imaging of the neck was done for patients with significant goiters, to rule out any retrosternal extension. Moreover, all patients underwent vocal cord function assessment and reported in the file before surgery.

Operative Technique: Operations were done under general anesthesia, endotracheal intubation, supine position 
with the fixed extended neck. A collar incision about $2 \mathrm{~cm}$ above the sternal notch. By using a monopolar cautery, a subplatysmal flap elevated superiorly and inferiorly. The midline of cervical fascia was dissected superiorly and inferiorly Once the thyroid gland is recognized, ligation of the middle thyroid vein, capsular blunt dissection at the lateral aspect of the thyroid lobe, the superior pedicle identified and then divided using either ties or Harmonic scalpel (Covidien, Mansfield, MA, USA). Blunt dissection or a Harmonic scalpel used to cut Berry's ligament, and all other fascial attachments. The parathyroid glands were identified and protected as well as the recurrent laryngeal nerves. After removal of the dissected thyroid gland/lobe, hemostasis, suction drain kept for 24 hours. Lastly, the wound closed in layers. Then, specimens were sent to the laboratory for Histopathology evaluation.

Statistical Analysis: Data presented as mean \pm SD, ranges, numbers, and ratios. Results analyzed using Wilcoxon's ranked test for unrelated data (Z-test) and Chi-square test ( $\mathrm{X}^{2}$ test) for numerical data. Statistical analysis conducted using the SPSS (version 19 for Windows; SPSS Inc., Chicago, Illinois, USA) statistical package. The $P$-value $<0.05$ was considered as statistically significant.

\section{Results}

The study included 100 patients; 22 males and 78 females with mean age $43.7 \pm 11.5$; range: $22-60$ years. Nodules were in the right lobe of the thyroid gland in 40 patients, in the left lobe in 32 patients, in the isthmus in 2 patients, and in both lobs in 26 patients (Table 1). The mean operative time in our study $105 \pm 17.9$; range: 55-150 minutes. Total thyroidectomy underwent for 48 patients, while hemithyroidectomy and near-total thyroidectomy were done for 37 and 15 patients respectively. The postoperative complications were minor with a transient hoarseness of voice and temporary hypoparathyroidism in $4 \& 6$ patients respectively (Table 2 ).

The Concordance Between TI-RADS and Bethesda System Categories: Fifty-five patients with thyroid nodules in our study were probably benign (TI-RADS 3 ), and FNA cytology established this result in $45 / 55$ patients revealing an $82 \%$ concordance rate between US and FNAC. Among the 31 cases that were categorized as TIRADS 4 (indeterminate), 8/31 cases were benign on FNAC (Bethesda II), 1/31 cases had a FNAC biopsy established as cancer, while the remaining 22/31 patients were fallen in Bethesda III, IV, and V giving a $70.9 \%$ concordance rate between the US and FNAC (see Fig. 3). Among the 14 cases that were categorized as TI-RADS 5 (malignant on the US), 2/14 patients were benign on FNAC biopsy (falsepositive impression of the US), 5/14 cases were suspicious on FNAC (Bethesda IV), and 7/14 cases had a FNAC biopsy established as cancer giving a $50 \%$ concordance between the US and FNAC. From the previous results, we can find that the overall concordance rate between US TIRADS and Bethesda is $67.6 \%$ (Fig. 3 \& 4)

The Concordance of TI-RADS and Bethesda system for the final PO Histological Diagnosis: The overall concordance rate of US TI-RADS with the final PO Histological results for predicting malignancy for predicting malignancy was $75.4 \%$ with a sensitivity of $76.9 \%$ and specificity $91.3 \%$, PPV 71.4\%, and NPV 76.4\%. On the other hand, the overall concordance rate of FNAC with the final PO Histological findings for predicting malignancy was $95 \%$ with a sensitivity of $81.8 \%$ and specificity $98 \%$, PPV 90\%, and NPV 96\%. (Table 3) summarizes the results and correlation.

Table 1: Patients' demographic data.

\begin{tabular}{|c|c|c|}
\hline Data & Strata & Findings \\
\hline Age (years) & & $43.7 \pm 11.5(22-60)$ \\
\hline \multirow{2}{*}{$\begin{array}{c}\text { Sex } \\
n(\%)\end{array}$} & Males & $22(22 \%)$ \\
\hline & Females & $78(78 \%)$ \\
\hline \multirow{2}{*}{$\begin{array}{c}\text { ASA score } \\
n(\%)\end{array}$} & $\mathrm{I} / \mathrm{II}$ & $82(82 \%)$ \\
\hline & III & $18(18 \%)$ \\
\hline \multirow{4}{*}{$\begin{array}{l}\text { Location of the nodule } \\
\qquad n(\%)\end{array}$} & Right lobe & $40(40 \%)$ \\
\hline & Left lobe & $32(32 \%)$ \\
\hline & Isthmus & $2(2 \%)$ \\
\hline & Bilateral & $26(26 \%)$ \\
\hline \multirow[t]{3}{*}{ Laboratory } & TSH (mlU/L) & $2.1+1.7$ \\
\hline & FT3 (pmol/L) & $5.1+3.5$ \\
\hline & FT4 (pmol/L) & $16.2+6.5$ \\
\hline
\end{tabular}

Data are presented as mean $\pm S D \&$ numbers; ranges \& percentages are in parenthesis. BMI: body mass index; ASA: American Society of Anesthesiologists; mIU/L: Milli-International Units per liter; pmol/L: Picomole per Liter; TSH: Thyroid-Stimulating Hormone; FT3: Free Triiodothyronine; FT4: Free Tetraiodothyronine. 
Table 2: Operative and PO collected data.

\begin{tabular}{|c|c|c|}
\hline Data & Strata & Findings \\
\hline \multirow{7}{*}{ Indications for Thyroidectomy } & Euthyroid nodular goiter & $28(28 \%)$ \\
\hline & Grave's disease & $8(8 \%)$ \\
\hline & Thyroiditis & $3(3 \%)$ \\
\hline & Follicular thyroid adenoma & $20(20 \%)$ \\
\hline & Follicular thyroid carcinoma & $5(5 \%)$ \\
\hline & Papillary thyroid carcinoma & $35(35 \%)$ \\
\hline & Medullary thyroid carcinoma & $1(1 \%)$ \\
\hline Operative time (mins) & & $105 \pm 17.9(55-150)$ \\
\hline Blood loss (ml) & & $60.5 \pm 15.4(50-160)$ \\
\hline \multirow{3}{*}{ Type of (Thyroidectomy) operation } & $\mathrm{HT}$ & $37(37 \%)$ \\
\hline & NTT & $15(15 \%)$ \\
\hline & TT & $48(48 \%)$ \\
\hline \multirow{2}{*}{ Postoperative complications* } & Transient Hoarseness of voice & $4(4 \%)$ \\
\hline & Transient Hypoparathyroidism & $6(6 \%)$ \\
\hline
\end{tabular}

Data are presented as mean $\pm S D$ \& numbers; ranges \& percentages are in parenthesis. PO: Postoperative; HT: Hemithyroidectomy; NTT: Near-total Thyroidectomy; TT: Total Thyroidectomy; *: more than one complication can happen in the same patient.

Table 3: Thyroid preoperative US result based on TI-RADS and FNAC results based on the Bethesda system with their correlation with the final postoperative Histology reports.

\begin{tabular}{|c|c|c|c|c|}
\hline \multirow{2}{*}{$\begin{array}{l}\text { Diagnostic } \\
\text { modality }\end{array}$} & \multirow{2}{*}{ Strata } & \multirow{2}{*}{ Number } & \multicolumn{2}{|c|}{ Final Histology } \\
\hline & & & Benign & \\
\hline \multirow{7}{*}{ Bethesda system } & Category & & & \\
\hline & II & 50 & $48(96 \%)$ & $2(4 \%)$ \\
\hline & III & 16 & $11(68.7 \%)$ & $5(31.3 \%)$ \\
\hline & IV & 17 & $8(47 \%)$ & $9(53 \%)$ \\
\hline & $\mathbf{V}$ & 7 & $3(43 \%)$ & $4(57 \%)$ \\
\hline & VI & 10 & $1(12.5 \%)$ & $9(87.5 \%)$ \\
\hline & Total & 100 & $71(71 \%)$ & $29(29 \%)$ \\
\hline \multirow{5}{*}{ TI-RADS } & Class & & & \\
\hline & TR3 & 55 & $42(76.4 \%)$ & $13(23.6 \%)$ \\
\hline & TR4 & 31 & $25(81 \%)$ & $6(19 \%)$ \\
\hline & TR5 & 14 & $4(28.6 \%)$ & $10(71.4 \%)$ \\
\hline & Total & 100 & $71(71 \%)$ & $29(29 \%)$ \\
\hline
\end{tabular}

Data are presented as numbers \& percentages are in parenthesis. TI-RADS: thyroid image reporting and data system. 


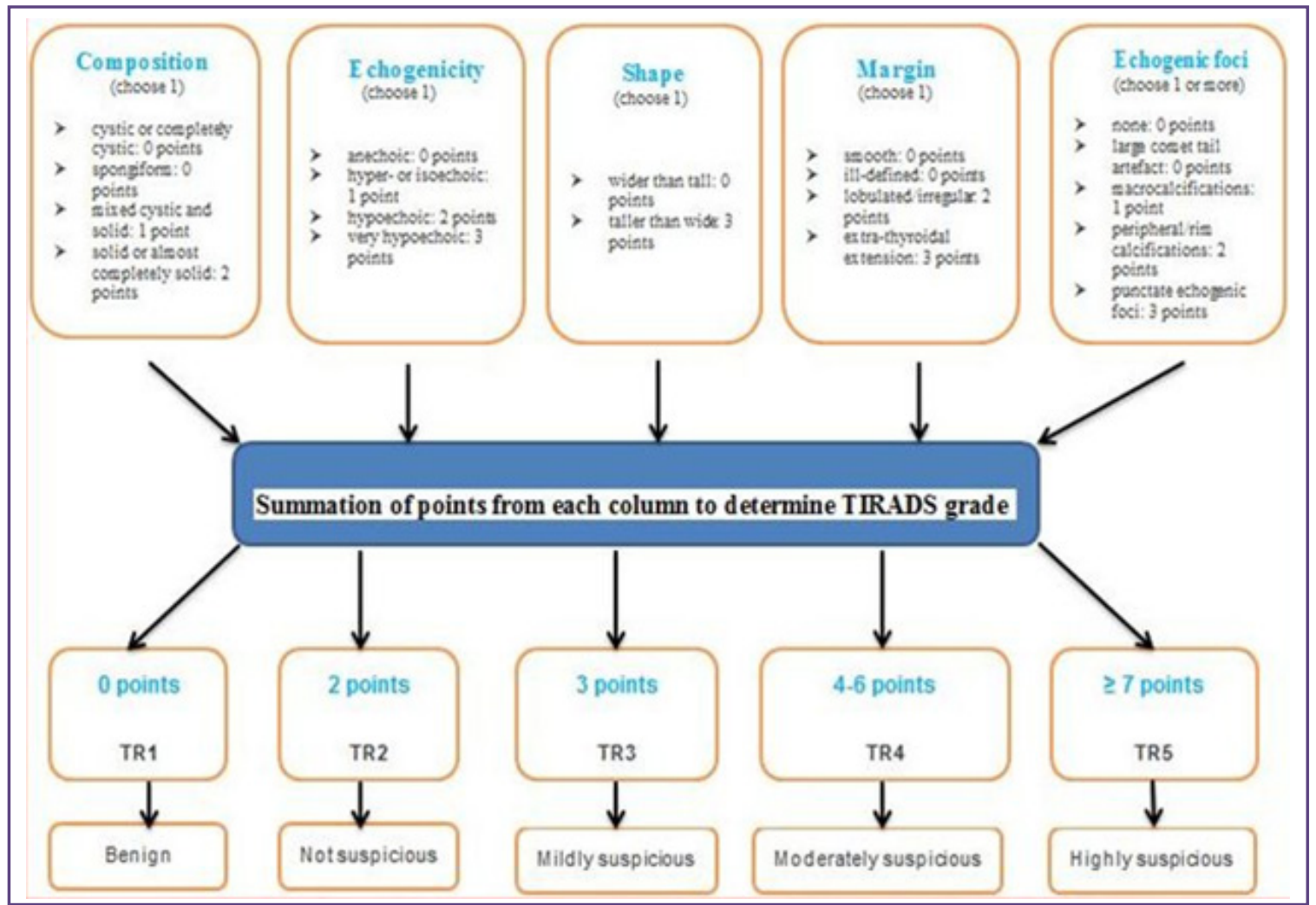

Fig. 1: TIRADS scoring depending on the cumulative points from the findings of afive US categories. (Source: Tessler et al 2017)

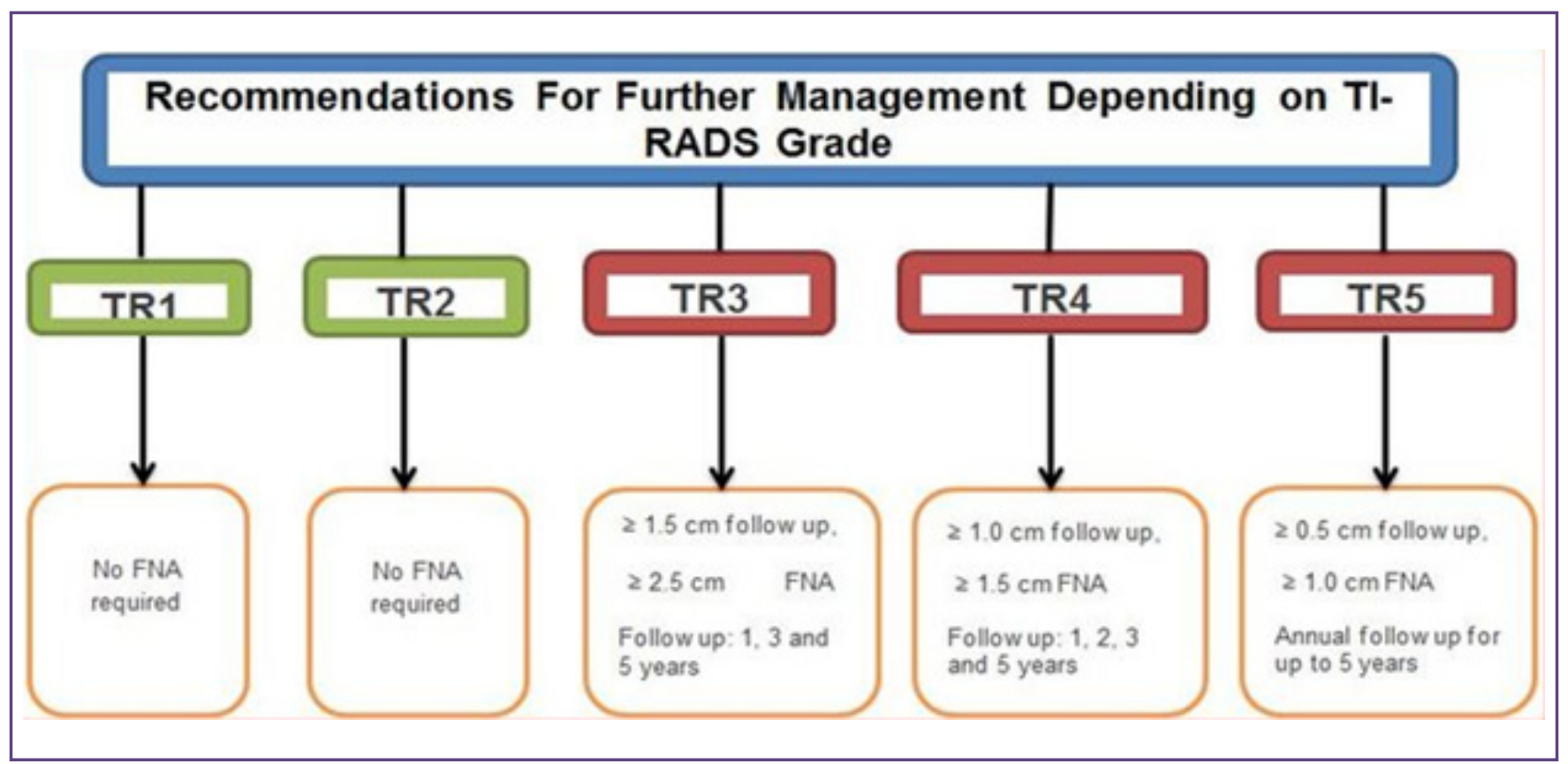

Fig. 2: Recommendations forthe further management of thethyroid nodulesdepending on the TI-RADS grade.

(Source: Tessler et al 2017). 


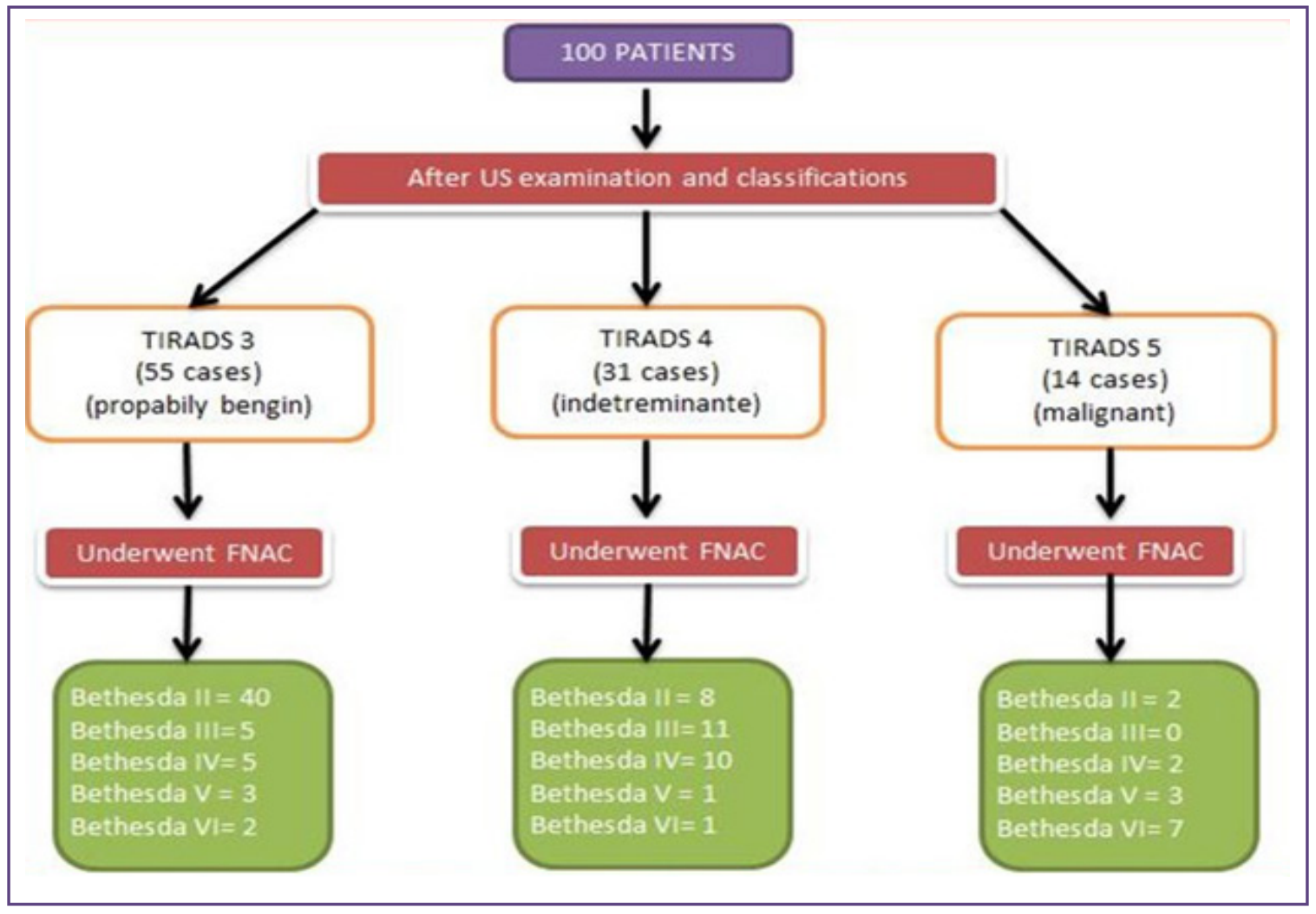

Fig. 3: The Results of (TI-RADS) classification in relation to (Bethesda)classification forthe evaluation of thyroid nodules.

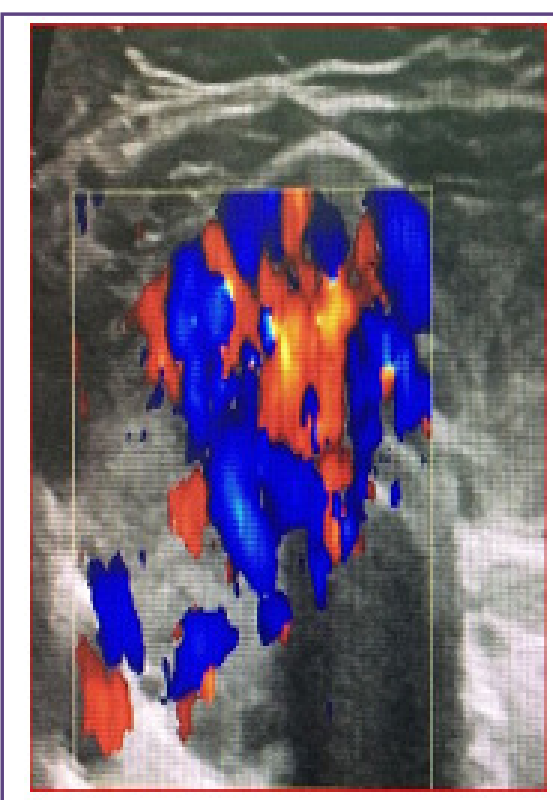

$\mathbf{a}$

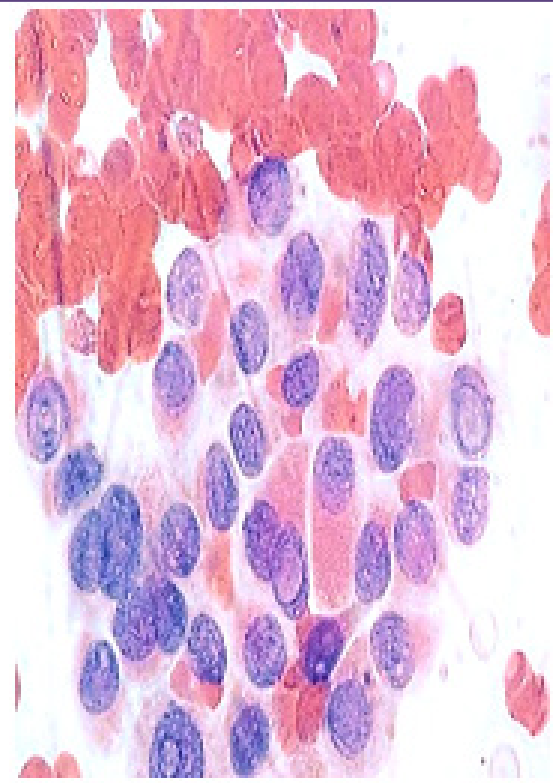

b

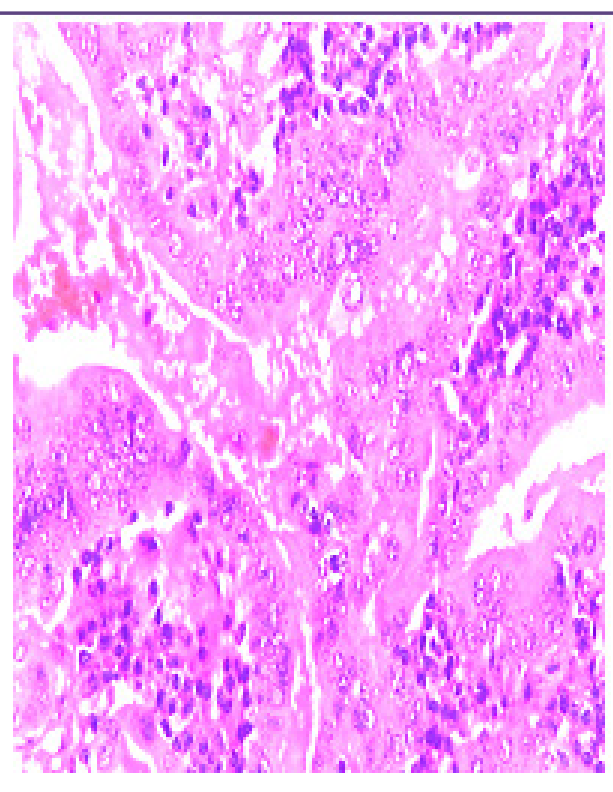

c

Fig. 4: a. Thyroid ultrasound showing an ill-defined thyroid nodule with hyper vascularity, b. FNAC of the thyroid nodule showing a nuclear enlargement, elongation, overlapping, grooving, and intra-nuclear cytoplasmic inclusion (H\&E $x$ 1000), and c.A Post thyroidectomy section of the same nodule showing papillae with a characteristic nuclear features of papillary thyroid carcinoma(H\&E x 400). 


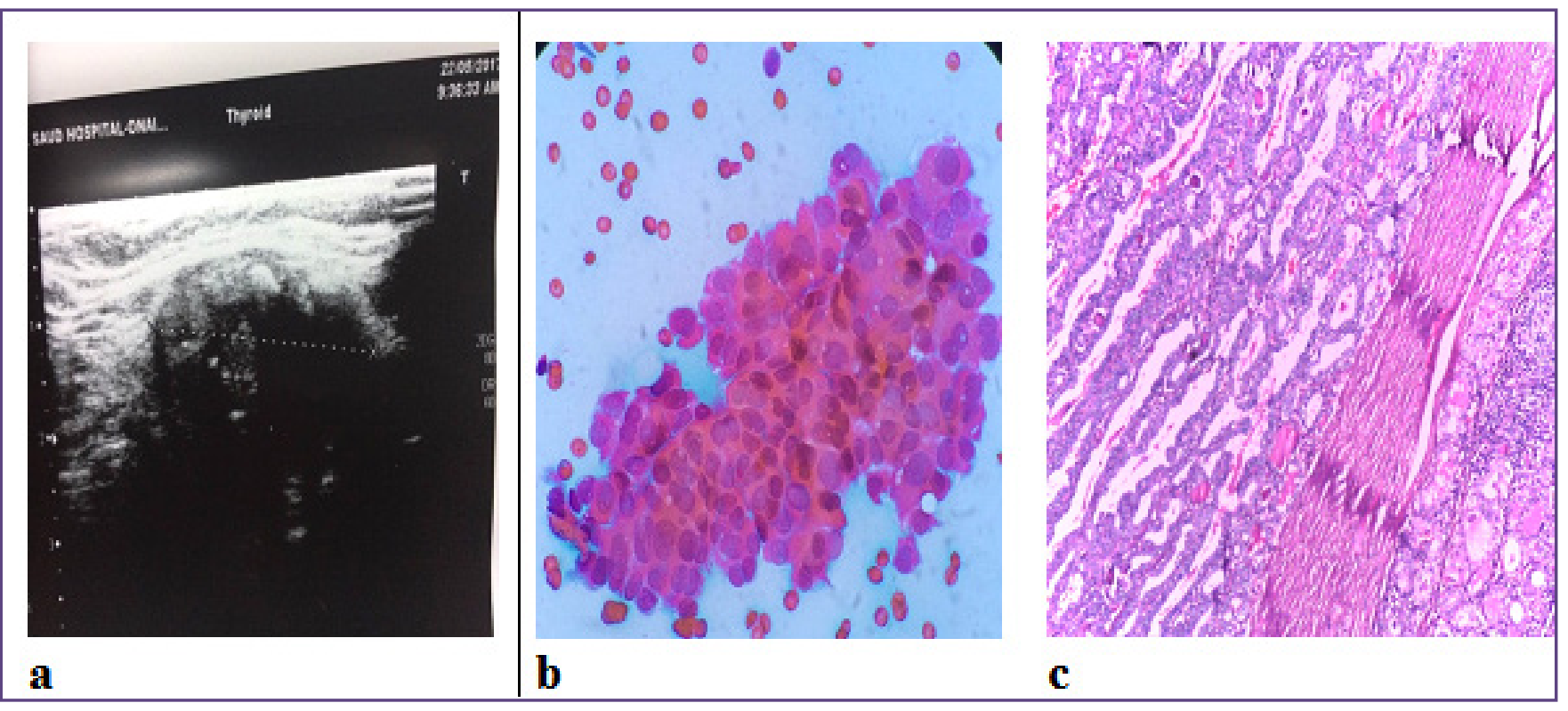

Fig. 5: a. Thyroid ultrasound showing a well-defined thyroid nodule with a heterogeneous echogenicity, b. FNAC of the thyroid nodule showing a crowded mono-layer sheet of a follicular cells arranged in a micro-follicular pattern (H\&E $x$ 400), diagnosed as follicular lesion of undetermined significance, and c. A Post thyroidectomy section of the same nodule showing a capsulated follicular variant of papillary thyroid carcinoma (H\&E x 100).

\section{Discussion}

Evaluation of a thyroid nodule disease based on a process of four-step (Quadruple assessment) including; (1) detailed history, plus clinical examination, (2) laboratory exams involving thyroid function tests, (3) thyroid US, and (4) US-guided FNAC based on TI-RADS features. ${ }^{[14]}$ It resembles the triple assessment of the breast lumps. However, TI-RADS US features are still not routinely used in several teaching institutes. ${ }^{[15]}$ The accessibility of TI-RADS and recent validation of this US classification system by the ACR permits a precise clinical and pathological correlation. As noted from the preceding studies, ${ }^{[16,17]}$ a strong clinicopathological correlation will guide us in defining the risk of malignancy and hence direct our proper management of thyroid lesions. ${ }^{[18]}$ In many countries around the world; surgeons, radiologists, and endocrinologists become aware of using the US classification system (TI-RADS) for evaluation of thyroid lesions and use it regularly in their clinical performance. ${ }^{[19]}$

Depending on our own analysis of 100 cases of thyroid lesions, we have seen a fairly good concordance rate of both US TIRADS and FNAC biopsy for the final PO Histological results that was $75.4 \& 95 \%$, respectively, which will be the baseline now for incoming quality upgrading as we become more conversant and experienced with the thyroid US. We have found from our experience in the outpatient clinic during the US evaluation of thyroid lesions that having a model of US pictures showing the variable TI-RADS categories are of benefit and help to diminish mistakes in recording the category of TI-RADS. Similar action is regularly used by endoscopists in the endoscopy unit for gastrointestinal lesions for example; grading of esophageal varices, reflux esophagitis, and bleeding gastric or duodenal ulcer.

When comparing our results with the outcomes of a study that was done by Singaporewalla et_al, $^{[20]}$ the US impression of thyroid nodules in our study had a comparable sensitivity in predicting malignancy $(71.5 \%$ versus $70.6 \%)$ but lower specificity (85\% versus $90.4 \%$ ). We also had a comparable NPV of TI-RADS score predicting malignancy (91.5\% versus $93.8 \%)$.

Regarding the overall accuracy, we had an accuracy of $75.3 \%$ compared with $83 \%$ in the previous mentioned study. On the other hand, on comparing our results with the data reported by Horvath et al, ${ }^{[7]}$ our US had a lower sensitivity in predicting malignancy in thyroid nodules $(71.5 \%$ compared to $88 \%)$ but higher specificity ( $85 \%$ compared to $49 \%$ ). We also had a higher NPV of TIRADS score predicting malignancy as well (91.5\% compared to $88 \%$ ).

There is no doubt that, using the TI-RADS system for US reporting permits clinicians to improve their US skills and track their results in comparison with the FNAC findings. In Singaporewalla et al study, ${ }^{[20]}$ they had 20 patients that were classified as TI-RADS 5 (malignant on the US), only 
12 were confirmed as malignant with FNAC biopsy and the remaining were benign. This gives them a $60 \%$ accuracy of US in predicting malignancy. Similarly, in our study, we found that among the 14 cases that were categorized as TIRADS 5, 10/14 cases had a FNAC biopsy established as cancer ( $71.4 \%$ accuracy).

The limitations of this study are that we did not define the clinic-pathological correlation of TI-RADS subgroups $4 \mathrm{a}, 4 \mathrm{~b}$ and $4 \mathrm{c}$ to the results of FNAC. Going forward, we hope to further sub-classify the TI-RADS 4 lesions into the different sub-categories and audit our results.

Several studies have verified the high global accuracy of FNAC biopsy for assessment of thyroid nodules, and this result has been established, mainly for patients with a solitary thyroid nodule and those with some US criteria of malignancy; however, FNAC biopsy has displayed less accuracy in the assessment of multinodular goiter. ${ }^{[21,22,23]}$ The overall accuracy of FNAC biopsy in comparison with the final PO Histological reports in our study was $95 \%$ with sensitivity and specificity of $81.8 \%$ and $98 \%$, respectively. In promise with these results, Krzysztof et al ${ }^{[24]}$ reported that the rate of prediction of thyroid carcinoma by FNAC in the patients with a thyroid nodule was $95.8 \%$ and it was higher in patients with a solitary nodule than that in patients with multiple nodules ( $98 \%$ versus $96 \%$, respectively) in the same study. ${ }^{[25]}$

\section{Conclusion}

Our present study reveals a respectable clinic-pathological correlation between the thyroid US that was reported by the TI-RAD classification system and the FNAC results of thyroid nodule using Bethesda classification. For surgeons, the use of the TI-RAD system as a risk stratification for classifying thyroid lesions will be adopted from this time in our practice throughout US assessment of thyroid lesions.

\section{Declarations}

The Authors declare that there is no conflict of interest

\section{References}

1. Vander J, Gaston E, and Dawber T: The significance of nontoxic thyroid nodules. Final report of a 15-year study of the incidence of thyroid malignancy. Ann Intern Med. 1968;69:537-554.

2. Tunbridge W, Evered D, Hall R, Appleton D, Brewis M, Clark F, et al: The spectrum of thyroid disease in a community: the Whickham survey. Clin Endocrinol (Oxf) 1977;7:481-449.

3. Mandel S: A 64-year-old woman with a thyroid nodule. JAMA. 2004;292:2632-2642.

4. Tan G, Gharib H. Thyroid incidentalomas: management approaches to nonpalpable nodules discovered incidentally on thyroid imaging. Ann Intern Med. 1997;126(3):226-231.
5. Gharib Hand Papini E. Thyroid nodules: clinical importance, assessment, and treatment. Endocrinol Metab Clin North Am. 2007;36(3):707-735.

6. Grant E, Tessler F, Hoang J, Langer J, Beland M, Cronan $\mathrm{J}$ et al: Thyroid ultrasound reporting lexicon: white paper of the ACR thyroid imaging, reporting and data system (TIRADS) committee. J Am Coll Radiol. 2015;12 (12 Pt A): 1272-1279.

7. Horvath E, Majlis S, Rossi R, Franco C, Niedmann J, Castro A, and Dominguez M: An ultrasonogram reporting system for thyroid nodules stratifying cancer risk for clinical management. J Clin Endocrinol Metab. 2009; 94(5):17481751.

8. Kwak J, Han K, Yoon J, Moon H, Son E, Park S, et al: Thyroid imaging reporting and data system for US features of nodules: a step in establishing better stratification of cancer risk. Radiology. 2011; 260 (3): 892-899.

9. Chng C, Kurzawinski T and Beale T: Value of sonographic features in predicting malignancy in thyroid nodules diagnosed as follicular neoplasm on cytology. Clin Endocrinol (Oxf). 2015; 83 (5): 711-716.

10. Yoon J, Lee H, Kim E, Moon H, and Kwak J: Thyroid nodules: non- diagnostic cytologic results according to thyroid imaging reporting and data system before and after application of the Bethesda system. Radiology. 2015; 276 (2): 579-587.

11. Naykky S, Juan P, Spyridoula M, Ana E, Rene R, Michael R, Ana C, et al: Diagnostic accuracy of ultrasound-guided fine needle aspiration biopsy for thyroid malignancy: systematic review and meta-analysis. Endocrine. 2016;53:651-661.

12. Tessler F, Middleton W, Grant E, Hoang J, Berland L, Teefey S, et al: ACR Thyroid Imaging, Reporting and Data System (TI-RADS): White Paper of the ACR TI-RADS Committee. J Am Coll Radiol. 2017; Vol.14, Iss. 5, P 587-595.

13. Cibas E and Ali S: The 2017 Bethesda System for Reporting Thyroid Cytopathology. 2017 Nov; 27(11):1341-1346.

14. Srinivas M, Amogh V, Gautam M, Prathyusha I, Vikram $\mathrm{N}$, Retnam M, et al: A prospective study to evaluate the reliability of thyroid imaging reporting and data system in differentiation between benign and malignant thyroid lesions. J Clin Imaging Sci. 2016; 6: 5.

15. Chandramohan A, Khurana A, Pushpa B, Manipadam M, Naik D, Thomas N, Abraham D and Paul M: (2016) Is TIRADS a practical and accurate system for use in daily clinical practice? Indian J Radiol Imaging. 2016; 26 (1): 145-152.

16. Hoang J, Langer J, Middleton W, Hammers L, Cronan J, Tessler F, Grant E, and Berland L: Managing incidental thyroid nodules detected on imaging: white paper of the ACR Incidental Thyroid Findings Committee. Journal of the American College of Radiology: JACR. 2015; 12 (2): 143-50.

17. Park J, Lee H, Jang H Kim H, Hyuck J, Lee W and Kim S: A proposal for a thyroid imaging reporting and data system for ultrasound features of thyroid carcinoma. Thyroid. 2009; 19 (11):1257-1264 
18. Moifo B, Takoeto E, and Tambe J: Reliability of thyroid imaging reporting and data system (TIRADS) classification in differentiating benign from malignant thyroid nodules. Open J Radiol. 2013; 3:103-107.

19. Hossein G, Enrico P, Jeffrey R, Daniel S, Mack H, Laszlo $\mathrm{H}$, et al: American Association of Clinical Endocrinologists, American College of Endocrinology, and Associazione Medici Endocrinologi Medical Guidelines for Clinical Practice for the Diagnosis and Management of Thyroid Nodules - 2016 Update. Endocr Pract. 2016;22(5):622-639.

20. Singaporewalla R, Hwee J, Lang T, and Desai V: Clinicopathological Correlation of Thyroid Nodule Ultrasound and Cytology Using the TIRADS and Bethesda Classifications. World J Surg. 2017; 41:1807-1811.

21. Grant E, Tessler F, Hoang J, Langer J, Beland M, Berland L, et al: Thyroid Ultrasound Reporting Lexicon: White Paper of the ACR Thyroid Imaging, Reporting and Data System (TIRADS) Committee. Journal of the American College of Radiology: JACR. 2015; 12 (12 Pt A): 1272-9.
22. Tollin S, Mery G, Jelveh N, Fallon E, Mikhail M, Blumenfeld $\mathrm{W}$, et al: The use of fine-needle aspiration biopsy under ultrasound guidance to assess the risk of malignancy in patients with a multinodular goiter. Thyroid. 2000;10:235-41.

23. Baloch Z, LiVolsi V, and Asa S: Diagnostic terminology and morphologic criteria for cytologic diagnosis of thyroid lesions: a synopsis of the National Cancer Institute Thyroid Fine-Needle Aspiration State of the Science Conference. Diagn Cytopathol. 2008;36(6):425-437.

24. Krzysztof K, Dorota D, Beata W, Marta S, Paweł D, Krzysztof S, et al: Fine-Needle Aspiration Biopsy as a Preoperative Procedure in Patients with Malignancy in Solitary and Multiple Thyroid Nodules. PLoS One. 2016; 11(1)

25. Coorough N, Hudak K, Jaumec JC, Buehler D, Selvaggi $\mathrm{S}$, Rivas $\mathrm{J}$, et al: Nondiagnostic fine-needle aspirations of the thyroid: is the risk of malignancy higher? J Surg Res. 2013;184:746-750.

*Corresponding author:

Ashraf M Abdelkader (MD, MRCS), Associate professor, General surgery department, Faculty of medicine, Benha University, EGYPT

Phone: +91 966592072034

Email: Dr_ashrafmahmoud@yahoo.com

Financial or other Competing Interests: None. 